Study protocol

\title{
Understanding the Relationship between Activity and Neighbourhoods (URBAN) Study: research design and methodology
}

\author{
Hannah M Badland ${ }^{1}$, Grant M Schofield ${ }^{1}$, Karen Witten ${ }^{2}$, Philip J Schluter ${ }^{3}$, \\ Suzanne Mavoa ${ }^{2}$, Robin A Kearns ${ }^{*}$, Erica A Hinckson ${ }^{1}$, Melody Oliver ${ }^{1}$, \\ Hector Kaiwai $^{5}$, Victoria G Jensen ${ }^{5}$, Christina Ergler ${ }^{4}$, Leslie McGrath ${ }^{1}$ and \\ Julia McPhee ${ }^{1}$
}

Address: ${ }^{1}$ Centre for Physical Activity and Nutrition Research, Auckland University of Technology, Auckland, New Zealand, ${ }^{2}$ Centre for Social and Health Outcomes Research and Evaluation, Massey University, Auckland, New Zealand, ${ }^{3}$ School of Public Health and Psychosocial Studies, Auckland University of Technology, Auckland, New Zealand and School of Nursing and Midwifery, The University of Queensland, Qld 4072, Australia, ${ }^{4}$ School of Geography, Geology, and Environmental Science, University of Auckland, Auckland, New Zealand and ${ }^{5}$ Whariki Research Group, Massey University, Auckland, New Zealand

Email: Hannah M Badland - hannah.badland@aut.ac.nz; Grant M Schofield - grant.schofield@aut.ac.nz; Karen Witten - k.witten@massey.ac.nz; Philip J Schluter - philip.schluter@aut.ac.nz; Suzanne Mavoa - s.mavoa@massey.ac.nz; Robin A Kearns* - r.kearns@auckland.ac.nz; Erica A Hinckson - erica.hinckson@aut.ac.nz; Melody Oliver - melody.oliver@aut.ac.nz; Hector Kaiwai - h.kaiwai@massey.ac.nz; Victoria G Jensen - v.g.jensen@massey.ac.nz; Christina Ergler - c.ergler@auckland.ac.nz; Leslie McGrath - lmcgrath@aut.ac.nz; Julia McPhee - julia.mcphee@aut.ac.nz

* Corresponding author

Published: 10 July 2009

BMC Public Health 2009, 9:224 doi:10.1 186/147/-2458-9-224

This article is available from: http://www.biomedcentral.com//47/-2458/9/224

(C) 2009 Badland et al; licensee BioMed Central Ltd.

This is an Open Access article distributed under the terms of the Creative Commons Attribution License (http://creativecommons.org/licenses/by/2.0), which permits unrestricted use, distribution, and reproduction in any medium, provided the original work is properly cited.
Received: 19 May 2009

Accepted: 10 July 2009

\begin{abstract}
Background: Built environment attributes are recognized as being important contributors to physical activity (PA) engagement and body size in adults and children. However, much of the existing research in this emergent public health field is hindered by methodological limitations, including: population and site homogeneity, reliance on self-report measures, aggregated measures of PA, and inadequate statistical modeling. As an integral component of multi-country collaborative research, the Understanding the Relationship between Activity and Neighbourhoods (URBAN) Study seeks to overcome these limitations by determining the strengths of association between detailed measures of the neighborhood built environment with PA levels across multiple domains and body size measures in adults and children. This article outlines the research protocol developed for the URBAN Study.
\end{abstract}

Methods and design: The URBAN Study is a multi-centered, stratified, cross-sectional research design, collecting data across four New Zealand cities. Within each city, 12 neighborhoods were identified and selected for investigation based on higher or lower walkability and Māori demographic attributes. Neighborhoods were selected to ensure equal representation of these characteristics. Within each selected neighborhood, 42 households are being randomly selected and an adult and child (where possible) recruited into the study. Data collection includes: objective and self-reported PA engagement, neighborhood perceptions, demographics, and body size measures. The study was designed to recruit approximately 2,000 adults and 250 children into the project. Other aspects of the study include photovoice, which is a qualitative assessment of built 
environment features associated with PA engagement, an audit of the neighborhood streetscape environment, and an individualized neighborhood walkability profile centered on each participant's residential address. Multilevel modeling will be used to examine the individual-level and neighborhood-level relationships with PA engagement and body size.

Discussion: The URBAN Study is applying a novel scientifically robust research design to provide urgently needed epidemiological information regarding the associations between the built environment and health outcomes. The findings will contribute to a larger, international initiative in which similar neighborhood selection and PA measurement procedures are utilized across eight countries. Accordingly, this study directly addresses the international priority issues of increasing PA engagement and decreasing obesity levels.

\section{Background}

Increasing physical activity (PA) engagement and reducing obesity levels at the population-level have been identified as national [1] and international [2,3] health priorities. Multiple factors at different levels, including personal, family, social, environmental, and economic attributes, have been shown to influence PA and obesity patterns [4]. Environmental determinants, such as changes in urbanization patterns and the built environment, increased used of labor-saving devices, greater participation in sedentary activities, and reliance on automobiles for transport are now being recognized as key contributors to these health outcomes [2,3,5]. Urban sprawl, a composite measure of many built environment elements, has also been positively related to populationlevel overweight/obese status [6-8], potentially through reduced accumulation of PA via increased reliance on cars and reduced access to local destinations and public transport infrastructure. Despite these emerging relationships, studies in this field often have methodological flaws that limit the robustness of the findings. More rigorous data are urgently required to inform decision-makers of the built environment variables with greatest potential for improving PA and obesity outcomes.

A number of built environment features have been consistently identified as promoting PA in both adults [9-11] and children $[12,13]$. For adults these include increased street network connectivity, higher residential population density, greater access to public spaces, shops, and services, and higher levels of mixed land use [14-17]. Adult PA levels are also influenced by streetscape characteristics including neighborhood aesthetics, green space, pedestrian infrastructure, and safety factors [16-20]. For children, distance to school [21], neighborhood design $[13,22]$, traffic safety [23], and access to green spaces [24] and recreation locations [12] have been associated with PA engagement.

Although this evidence is accumulating, there are limitations in many of the studies on which it is based. To date, the majority of research investigating built environment variables with PA engagement and body size has relied on self-report measures, largely drawn from adult samples based in the United States and Australia. Although selfreport measures are practical to implement, they do not accurately detect incidental PA accumulation [25] and are affected by recall bias in adults [25] and children [26]. Also, neighborhoods have often not been selected to maximize variation in built environment attributes. Capturing neighborhood variability is fundamental to understanding the magnitude of built environment effects on individual PA engagement within these communities.

Ethnic differences in relation to built environment variables have also been understudied. Within New Zealand, Māori (New Zealand's indigenous people) have higher obesity rates when compared with New Zealand Pākehā/ European [27], and it is unknown whether urban form characteristics may influence these groups differently. Furthermore, little research has been conducted with children in this context; yet given the more sporadic nature of PA engagement displayed by children when compared with adults, it is conceivable that built environment variables associated with PA and body size will differ for adults and children. Parental perceptions of neighborhood safety (e.g., stranger danger, traffic concerns) may also be an important influencing factor regarding children's PA engagement [23]. Ignoring these important variables is likely to have resulted in population and site homogeneity, which in turn, may lead to underestimation of effect sizes in associations between the built environment and health outcomes. A further limitation of existing research in this field has been the use of rudimentary analytical techniques that ignore clustering and the multilevel or hierarchical structure of data on individuals living in different households, neighborhoods, cities, and countries. Multilevel modeling that can simultaneously account for factors at individual and neighborhood levels is likely to provide a more robust and sophisticated understanding of PA and health determinants [28].

The International Physical Activity and Environment Network (IPEN) study was set up to overcome the limitations 
inherent with many previous studies and to address the paucity of rigorous scientific evidence available in this field (refer http://www.ipenproject.org). Key strengths of the collaborative study are the: multi-country participation (Australia, Belgium, Colombia, Czech Republic, Hong Kong, New Zealand, United Kingdom, United States of America) to ensure inclusion of diverse urban environments, and the use of standardized protocols to measure the built environment (geographical information systems (GIS)), PA engagement (International Physical Activity Questionnaire - Long Form (IPAQ-LF), accelerometry), and other health outcomes (body size). Once collected, participant and neighborhood data will be combined to facilitate intra-and inter-country multilevel comparisons of built environment, PA, and health outcomes. This will produce more accurate effect size estimations, and improve understanding of international associations between the urban design, PA, and body size status. Purposefully stratifying neighborhoods based on built environment attributes and combining data from multiple sites in diverse countries will ensure that a larger variation of environmental attributes will be gained than those available from any one country.

The Understanding the Relationship Between Activity and Neighbourhood (URBAN) Study contributes to this collaboration by collecting New Zealand-specific built environment and health data from four diverse cities in accordance with IPEN protocols. In addition to the design strengths of the IPEN collaboration, the URBAN Study has incorporated several additional features that will add to its potential to contribute to understanding in this field: a child sample, stratifying neighborhoods by walkability and ethnicity, door-to-door recruitment of participants, streetscape audits, in depth assessments of the perceived environment (via photovoice), and individualized walkability profiles based on participants' residential location. This paper outlines the methods developed for use in the URBAN Study.

\section{Methods \\ Study aim}

The overarching aim of the URBAN Study is to understand the associations between neighborhood built environment variables, PA engagement, and body size. Measures of neighborhood urban design, PA levels across multiple domains (leisure, transport, habitual, and overall), and body size will be used to model the associations and establish effect sizes in a diverse sample using apposite statistical modeling.

\section{Study design}

This research is a cross-sectional study that examines the associations between neighborhood urban design, PA levels, and body size in adults and children residing in selected neighborhoods within four cities in New Zealand (North Shore, Waitakere, Wellington, and Christchurch). The sites were selected for their geographical diversity and because of existing access to city-level GIS data. The study was conceptualized using a multilevel framework, with the levels being: country, city, neighborhood, household, and individual. The URBAN Study is being conducted in seven phases, where each phase informs the subsequent stages of the research (Figure 1). Recruitment for the URBAN Study commenced in April 2008 in North Shore City, and the project uses a rolling data collection process across the four cities; it is anticipated that it will take one and a half years to complete the door-to-door data collection component of the study (phase 4). The host institutions of the research granted ethical approval for the outlined study procedures (AUTEC: 07/126, MUHECN: 07/045).

\section{Phase I: Neighborhood stratification}

Within each of the four cities, 12 neighborhoods were selected for investigation. In order to select neighborhoods, a walkability index was created and the domiciled Māori population was estimated. These values were applied to each mesh-block within the cities' boundaries. A mesh-block is a geographic census unit of approximately 100 households constructed by Statistics New Zealand [29]. The walkability index was calculated using combined measures of street connectivity, dwelling density, land use mix, and retail floor area ratio, and was generated using GIS software, ArcInfo 9.1 (ESRI, Redlands, $\mathrm{CA})$. The construction of these measures replicates existing IPEN research procedures $[10,30]$. Each of the walkability variables is discussed below.

\section{- Street connectivity}

Street connectivity was estimated by calculating intersection density. Street intersections with three or more unique intersecting streets were extracted from road network data. Mesh-block boundaries are typically defined by street centerlines. Therefore, to ensure that street intersections coincidental with mesh-block boundaries were included, intersection density was calculated as the number of intersections per square kilometer within 20 meters of each mesh-block boundary. Values for each mesh-block were between 0 and 1 , where a score closer to 1 indicated higher street connectivity.

\section{- Dwelling density}

The number of dwellings was estimated using mesh-block data for the number of occupied private dwellings taken from the New Zealand 2006 census [29]. Residential land area was obtained from the land use and zoning data provided by the territorial authorities. Dwelling density was calculated by dividing the number of dwellings by the residential land area for each mesh-block. 


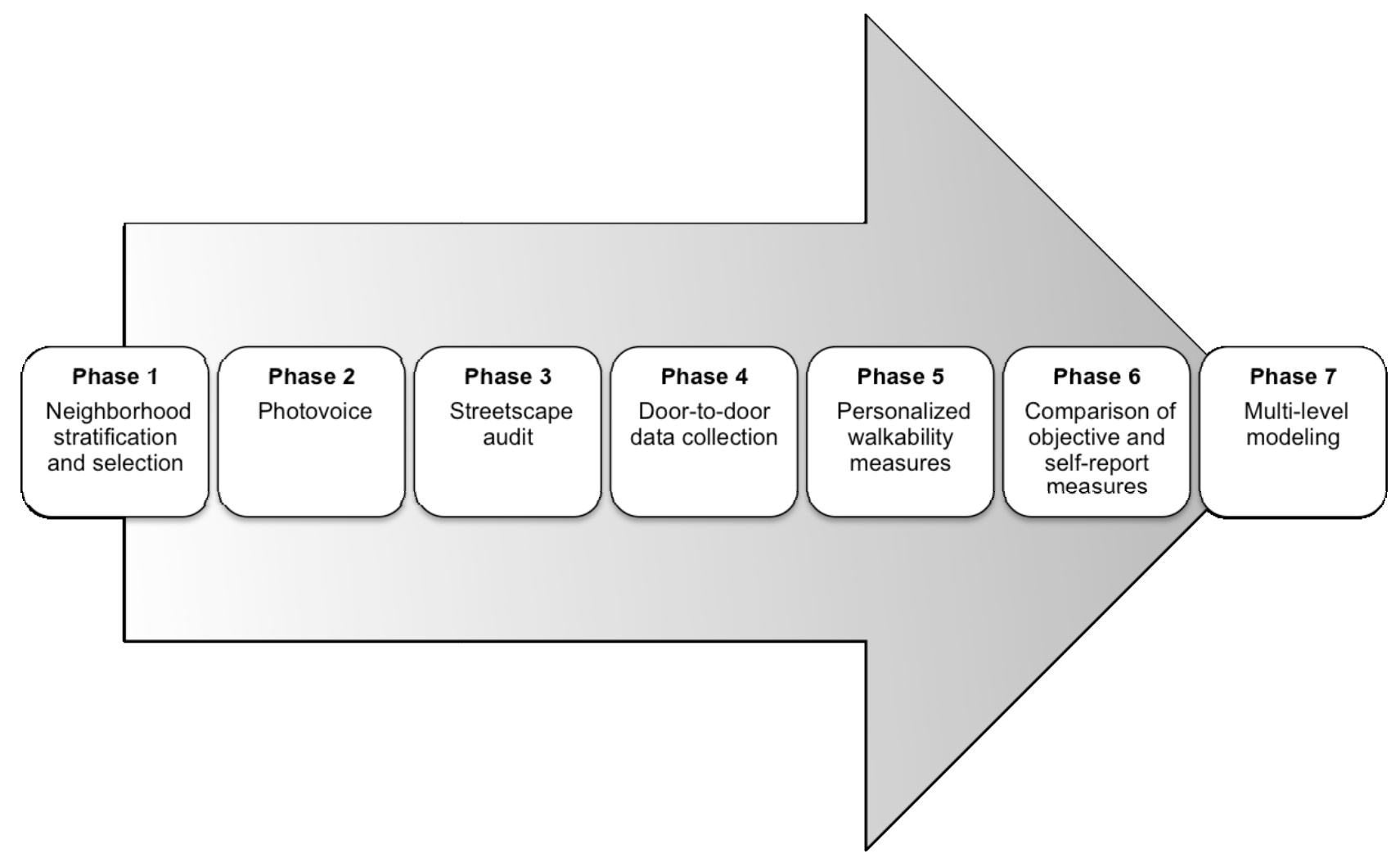

Figure I

Diagram of the overall research design for the URBAN Study.

- Mixed land use

The land use and zoning data were used to categorize land uses into commercial, residential, industrial, open space, and other within each mesh-block. The land use mix was calculated using an entropy index [31], where 0 indicates homogeneity of land use, and a value closer to 1 specified greater heterogeneity of land uses.

\section{- Retail floor area ratio}

The retail floor area was determined by using building outline data sourced from the territorial authorities. The net retail area was then calculated by dividing the retail floor area by the total retail parcel area within each meshblock [10]. A higher value indicated less parcel space allocated to car parking at retail sites within the mesh-block.

\section{- Walkability index}

The walkability index was calculated separately for each city using the above four measures (street connectivity, dwelling density, mixed land use, and retail floor area ratio). The measures were classified into deciles and recoded into values from 1 ( $1^{\text {st }}$ decile) to 10 ( $10^{\text {th }}$ decile). The walkability index for each mesh-block was calculated by summing the four 1 to 10 scores, resulting in a possible score from 4 to 40 .

\section{- Māori population}

Distribution of usual Māori residents domiciled within each mesh-block within the four cities was estimated by using 2006 census data [29]. Following the walkability index procedures, the mesh-block Māori population density was classified into deciles and recoded into values from 1 ( $1^{\text {st }}$ decile) to 10 ( $10^{\text {th }}$ decile) for each city. Māori comprise $14.6 \%$ of the resident population. They are the second-largest ethnic group (after New Zealand Pākehā/ European) in New Zealand [32].

\section{- Neighborhood selection}

Within each city, walkability and Māori population density were each partitioned into tertiles (lower (deciles 13), middle (deciles 4-7), and higher (deciles 8-10)). In the interest of capturing variability, only mesh-blocks with higher walkability and higher Māori population density, higher walkability and lower Māori population density, lower walkability and higher Māori population density, and lower walkability and lower Māori population density were eligible for this study. The middle tertile was removed from further analysis at this point.

All eligible mesh-blocks were then identified on city maps and clusters of five contiguous mesh-blocks of similar 
walkability and/or Māori population density characteristics were grouped together to form neighborhoods. The research team then purposefully selected three neighborhoods for each walkability/Māori population strata per city. This ensured geographical spread within each region and diversity across cities were captured. In total, 12 neighborhoods were selected per city and 48 neighborhoods were chosen across New Zealand. All neighborhoods are drawn from urban settings. In the instances where the number of potential respondents is exhausted within the neighborhood during the door-to-door recruitment phase (generally because of a high number of commercial premises within that setting), an additional contiguous mesh-block of similar built environment and Māori population characteristics is added to the neighborhood.

\section{Phase 2: Photovoice}

Photovoice is a research method that allows individuals, including those who may be marginalized, to conceptualize their environment through photography. In this study, neighborhood features associated with PA engagement across different cities, settings, and populations are qualitatively captured by photovoice. Children, as well as adults (approximately $\mathrm{n}=10$ per group) are drawn from five diverse neighborhoods in North Shore and Waitakere cities and invited to participate in the photovoice component of the URBAN Study. These participants do not necessarily partake in the door-to-door data collection aspect of the study. After an initial briefing, participants are each provided with a disposable camera to take photographs of features in their local environment they perceive make their self-defined neighborhood more and less conducive for engaging in PA. The photographs are developed, brought to a participant focus group (either adult- or child-specific). Participants presented noteworthy photos in relation to neighborhood PA attributes and explained the images to the group, both verbally and by way of captions written underneath the pictures. This process, either in small breakout groups or as a whole group discussion, enables the identification of key PA themes of significance and concern for participants in each locality. The discussions are audio taped, transcribed, and thematic induction analyses is conducted using Nvivo software (QSR, VIC, Australia). The photovoice procedures follow an established methodology [33,34].

\section{Phase 3: Streetscape audit}

In 12 selected street segments in each study neighborhood a streetscape audit using a modified version of the Systematic Pedestrian and Cycling Environment Scan (SPACES) tool [35] is undertaken to assess the presence and absence of features that support walking and cycling (e.g., physical infrastructure, aesthetics, traffic safety attributes). The SPACES, developed in Australia, has demonstrated appro- priate reliability for most variables examined in that setting (kappa $\geq 75 \%$ agreement) [36], and was adapted for the New Zealand context. The starting point for the audit is randomly selected within the neighborhood and thereafter the street segments are selected sequentially. Scores from each street segment are combined to provide a neighborhood streetscape value. All streetscape audits are conducted when door-to-door data collection is occurring in the city. For reliability purposes, $10 \%$ of the street segments are re-audited by a second trained assessor. A training manual based on the SPACES protocols was developed for the URBAN Study that included New Zealand specific reference images.

\section{Phase 4: Participant recruitment and data collection}

Trained interviewers recruited participants using a doorto-door recruitment strategy. For each selected neighborhood, GIS is used to generate street maps, identify parcel lots, random start points, walk paths, and enumerate households. These maps are provided to three or four trained interviewers for door-to-door recruitment with instructions to approach every $\mathrm{n}^{\text {th }}$ household. The household sampling rate is determined by dividing the neighborhood dwelling density [29] by the estimated response rate of $60 \%$. This value varies between neighborhoods because of the changeable number of residential dwellings located within each mesh-block. Commercial or temporary residential (e.g., motel rooms) premises are excluded from the sampling frame.

Interviewers start from GIS-derived randomly selected start points and approach the households identified by the enumeration process. The interviewers follow the predetermined walk path for each neighborhood. Forty-two households are selected in each neighborhood, and one adult and one child (where possible) are surveyed per household. This sampling strategy is designed to yield a total of 2,000 adult participants once data collection is complete. It is estimated that 250 children will be recruited into the study.

Individuals aged between 20-65 years and 3-12 years inclusive usually resident in private dwellings in the 48 selected neighborhoods are eligible to participate in the study. Where there is more than one eligible person in the household, potential participants are identified by the criterion of having the next birthday. Exclusion criteria are: falling outside the age ranges, not intending on living in the household over the measurement period, not resident in the dwelling three months prior to recruitment, unable to speak the English language, or having walking mobility restrictions, such as using crutches. The eligible child in the household is unable to participate in the research if the eligible adult from the household refuses to take part in the study. In the event that there is no eligible adult 
residing in the household or the eligible adult refuses to participate, the household becomes 'closed' and the interviewer moves on to the next household identified on the neighborhood walk. If no one is at home or an eligible adult resides in the household, but is not available, the interviewer makes a maximum of five return visits for recruitment purposes. The outcome for each visit is recorded on a door-to-door call sheet. Information regarding door-to-door recruitment procedures is documented in a training manual and briefing session.

Once participants are recruited, two data collection points (data collection 1, data collection 2) are arranged eight days apart, providing a seven-day measurement period (Figure 2). At data collection 1 the interviewer introduces the study, gains informed consent/assent, and distributes the accelerometer and travel/compliance log. Data collection 1 is frequently undertaken at the point of participant recruitment. The interviewer telephones the adult participants three days after data collection 1 to monitor accelerometer compliance. At data collection 2, the interviewer collects the accelerometer and travel/compliance log, measures participants' height, weight, waist and hip cir- cumferences, and conducts the survey with the adult participant. The interviewer follows the same call back recruitment procedures if the participant is not home for data collection 2 .

The interviewer enters all information directly on to a personal digital assistant at both time points and subsequently exports the data into Microsoft Excel (Microsoft Corp, Redmond, WA) at the research centre. Quality control audits are conducted on $10 \%$ of all interviews by the fieldwork supervisor.

\section{- Objective PA measures}

PA is measured objectively in adults and children for seven consecutive days using hip-mounted Actical accelerometers (Mini-Mitter, Sunriver, OR) fitted to a purposebuilt elastic waistband. The units have been shown to be reliable and valid for these populations [37-39]. Prior to distribution, the accelerometer supervisor prepares the units, including date stamping the devices and setting up the units to record PA and step counts in 30-second epochs. Accelerometers are distributed at data collection 1 by the interviewer and participants are instructed to wear

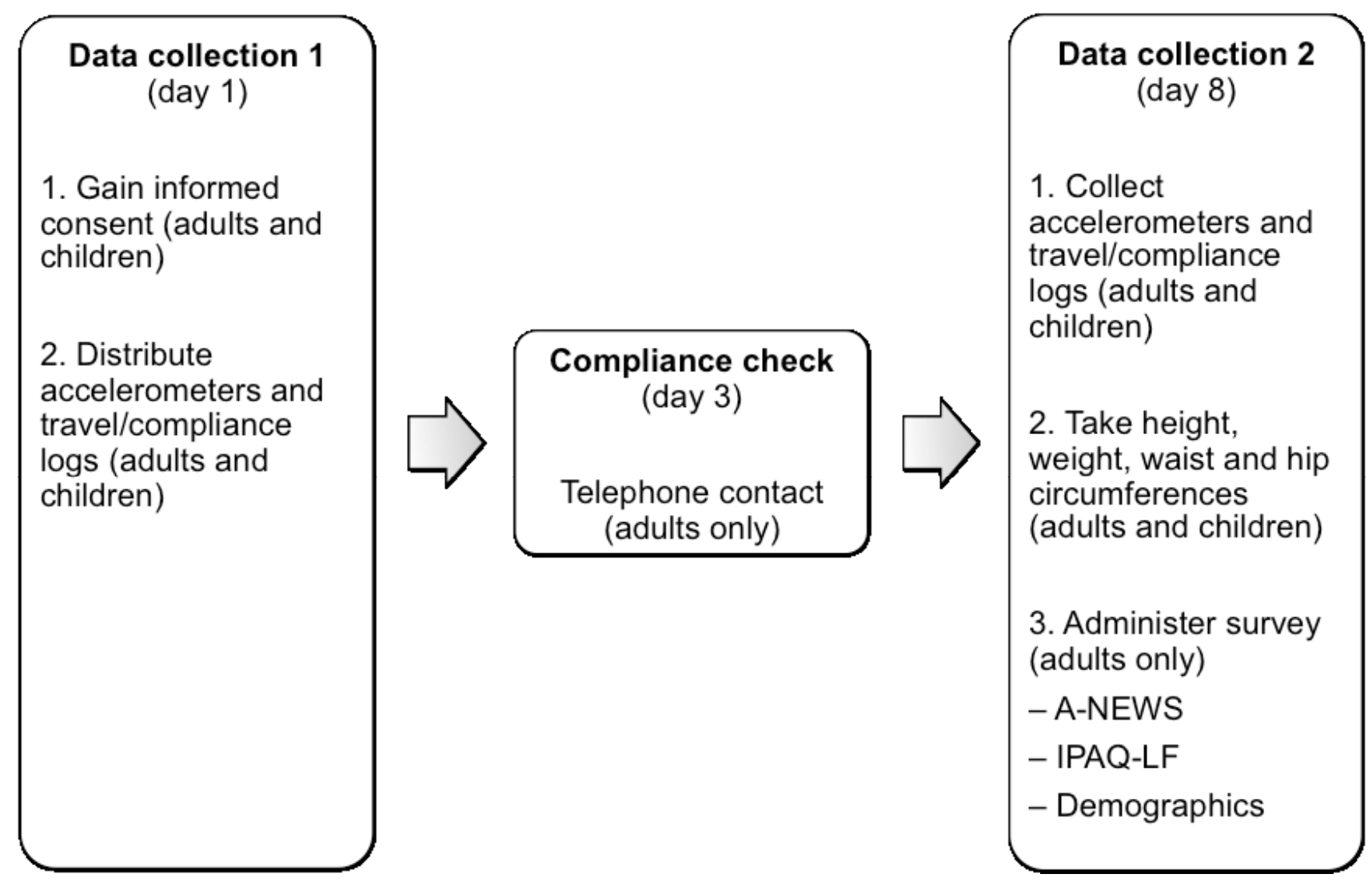

Figure 2

Measurement battery for the door-to-door component of the URBAN Study. 
the units for all waking hours for one week (seven days), but remove the monitors when participating in waterbased activities. Accelerometers are collected as close as possible to eight days later at data collection 2 by the interviewer. Accelerometers are returned to the research centre and the data are downloaded into Microsoft Excel by the accelerometer supervisor. Once cleaned, data from the unit are included for further analyses if at least 10 hours of data are gathered per day, for a minimum of 5 days. This is in accordance with IPEN protocols [40]. A manual has been developed regarding accelerometer downloading and uploading procedures, data storage, and data cleaning treatment protocols, and automated data extraction procedures are currently being developed. For most analyses, the outcome variables for adults and children will be the percentage of waking time spent in sedentary, light, moderate, and vigorous PA [41].

\section{- Travel/compliance log}

Participants self-complete a travel and compliance log for the duration of the accelerometer data collection. Each day, participants record what transport mode(s) they use to travel to and from work or study, the times they get up and go to bed, whether the accelerometer is removed for portions of the day, and if so, what activities the participant engages in during those times. No reliability or validity testing has been conducted with this tool. The information on waking hours and accelerometer removal derived from the log are checked and matched against accelerometer data.

\section{- Body size measures}

The interviewer measures body size at data collection 2 . Height is assessed to the nearest $0.1 \mathrm{~cm}$ using a stadiometer (Mentone Educational Centre, Victoria, Australia) and weight to the nearest $0.1 \mathrm{~kg}$ using calibrated Seca 770 scales (Protec Solutions Ltd, Wellington, NZ). Body mass index (BMI) status for adults will be determined using the World Health Organization ethnic-specific thresholds $[42,43]$ and the International Obesity Task Force criteria [44] will be applied to children. Waist circumference is measured as the minimum value between the iliac crest and the lateral costal margin (the mid-point between the hip and the lowest rib) to the nearest $0.1 \mathrm{~cm}$ using a Lufkin W606PM tape (Cooper Tools, Apex, NC, USA). Hip circumference is measured at the widest part of the buttocks [45]. Age-specific thresholds for high trunk mass will be applied to the sample [46-48].

\section{- Neighborhood perceptions}

Neighborhood perceptions are assessed using the Abbreviated-Neighborhood Walkability Scale (A-NEWS). The A-NEWS is a 54-item tool that measures adults' perceptions of dwelling density, land use mix, street connectivity, walking and cycling infrastructure, safety, and access to public and private facilities within their self-defined neighborhood. Responses are rated either on a four- or five-point Likert scales. Acceptable reliability and validity of the A-NEWS has been determined previously [49]. Neighborhood self-selection preferences are assessed on a five-point Likert scale using six items taken from the Strategies for Metropolitan Atlanta's Regional Transportation and Air Quality Study [50]. The neighborhood self-selection measures have also been used in the Neighborhood Quality of Life Study [51] and the Physical Activity in Localities and Community Environments [9]; these studies also contribute to the IPEN dataset.

\section{- Self-reported PA}

The IPAQ-LF is administered to capture adults' selfreported PA levels for the previous seven days (the period when the accelerometer was worn). The IPAQ-LF has shown to be a reliable and valid measure of PA engagement in 12 countries [40], and is used to assess PA engagement across four domains: occupational, transportation, household, and leisure. The outcome measures for overall and domain-specific PA will be frequency (days), duration (minutes), and intensity (light, moderate, and vigorous) of engagement. Self-reported PA levels will be compared with national PA recommendations, accelerometer data, and other countries participating in IPEN.

\section{- Demographics}

As part of the study, adult participants complete a demographic survey that examines: ethnicity, marital status, household income, academic qualifications, occupation, travel mode engagement, dwelling type, number of children living in the dwelling, time spent watching television, perceptions of body size, and the location of proximal and usually accessed food stores. Adult participants also complete the child's survey by proxy if an eligible child within the household participated in the study. Questions relating to the child include: ethnicity, screen time (e.g., television, computer, games consoles) access and rules, PA participation and motor skill ability, perceptions of body size, and access to and use of potential PA settings.

\section{- Weather}

Daily weather data (minimum and maximum temperature $\left({ }^{\circ} \mathrm{C}\right)$, rainfall $(\mathrm{mm})$ ) are recorded at sites located in each of the four cities. The New Zealand Metrological Service collects and provides this information. Timematched weather variables will be created to examine or control for the weather effects on PA engagement.

\section{Phase 5: Personalized walkability measures}

Personalized walkability index values will be calculated and constructed for adults and children based on the physical environment surrounding each participant's 
place of residence. The buffer distance will be developed along a one-kilometer street network from the participant's residence, excluding areas that cannot be accessed due to major barriers (e.g., freeways, water features). Similar GIS approaches as used to construct the walkability indices applied to the neighborhood selection process will be used to create the personalized walkability index classifications. Other potential inclusions in the index include public open space, public transport infrastructure, and topography variables within the buffer zone. Creation of these individualized measures has been conducted in previous research [52-54], and is a useful tool to enable the objectively measured built environment variables to be compared with individual-level health and self-report data.

\section{Phase 6: Comparison of objective and self-report measures} International research suggests there is a mismatch between measures of perceived and objectively assessed PA facility availability [55] and behaviors [56], and these relationships require further investigation. Accordingly, it is important to examine the independent associations and levels of agreement between actual and perceived PA infrastructure at the neighborhood level within the New Zealand context, and the relationships between objective and perceived PA behaviors. Objective measures derived from GIS, the streetscape audit, accelerometers, and body size will be compared with self-report measures drawn from the photovoice and door-to-door data collection components of the study.

\section{Phase 7: Multilevel modeling}

Multilevel modeling is one of the more appropriate methods for understanding how multiple factors occurring at various hierarchical levels (such as individual, household, neighborhood, and city variables) operate to influence PA engagement and body size. The sampling frame and research design enables multilevel analyses of neighborhood environmental predictors for self-reported and objectively measured PA and body size for Māori and non-Māori adults and children. These analytic strategies appropriately accommodate and model the hierarchy and clusters within the research design, and allow for the adjustment of important potential confounders (such as rainfall). Further analyses will likely consider how the influence of parental variables impacts on child health behaviors at the household level.

\section{Power calculations}

Precise power calculations depend on focused and predetermined statistical quantities; something that can be difficult for multi-aimed and broad studies such as this. For the purpose of this study, we intend recruiting 2,000 adults. However, a $10 \%$ reduction of our data is expected due to lack of compliance, reducing the data available for full analysis to 1,800 adults. Based on 12 background covariates explaining $25 \%$ of the variability of the dependent variable, and intraclass correlation coefficient cluster effects of 0.05 , a realized sample of 1,800 adults, $\alpha=0.05$ and statistical power of $80 \%$, the clustered multi-linear regression models will detect the smallest change in $r^{2}$ of $\leq 2.3 \%$ and clustered logistic regression models odds ratio of $\leq 1.27$ if the prevalence rate of overweight/obesity is $60 \%$. For the Māori and non-Māori comparisons, we expect lower Māori neighborhoods to have approximately $7 \%$ of the usual residents classified as Māori and higher Māori neighborhoods to have approximately $30 \%$ of the usual residents to identify as Māori [32]. Assuming a significant level of $\alpha=0.05$ and statistical power of $80 \%$, then the detectable difference between any Māori and non-Māori proportion is within $\pm 10 \%$ for this sample size. A difference of $\pm 10 \%$ was considered epidemiologically worthwhile and important to detect.

\section{Discussion}

Although characteristics of the built environment have been related to PA engagement $[9,11,12,22]$ and obesity levels [6-8], the epidemiological understanding of the associations between urban form and health outcomes still remains largely unknown. Improved understanding of built environmental influences on health behaviors, through socio-ecological models, is needed to inform more effective and sustainable interventions [28]. The URBAN Study will contribute to the evidence base pertaining to PA engagement, body size, and the built environment for adults and children by overcoming some of the existing methodological limitations in this field.

\section{Applications of the URBAN Study}

Four key research gaps in this area have been identified which the URBAN Study attempts to address. First, it is feasible that the limited environmental variability shown in urban locations previously investigated has underestimated the strength of associations between health outcomes and urban design [28]. The URBAN Study purposefully selects neighborhoods based on a diverse range of walkability and ethnicity characteristics, and contributes data to a multi-country study (IPEN). Second, although several studies have documented associations between the built environment and weight status $[6,8]$ and PA engagement $[57,58]$, confirmatory studies have yet to be conducted in diverse communities using robust measures to determine any walkability effect. Understanding these relationships in greater detail using standardized objective measurement procedures and protocols (GIS, accelerometers, body size) will provide more rigorous urban planning guidance to decision makers, thereby increasing the likelihood of improving population-level body size and PA outcomes. To our knowledge, this is the first New Zealand study to simultaneously use objective 
and self-report measurement tools to assess adult and child PA levels and body size status with the built environment. Third, limited evidence exists regarding how those individuals of different ethnicities, ages, genders, and/or family structures are influenced by the impact of neighborhood design with regard to health outcomes [28]. The URBAN Study has been designed to in part address this issue, with findings that can be stratified and analyzed according to these variables. Fourth, internationally there is very little evidence available identifying which built environment variables influence children's PA and body size, and how the built environment impacts on parental choices regarding children's PA opportunities. Accordingly, the URBAN Study will contribute directly to this evidence base by examining the interactions between children's PA behaviors, body size, parental perceptions, and built environment characteristics across diverse settings and child age ranges. It is anticipated that full results of the study will be available in 2011 .

\section{Strengths and weaknesses of the URBAN Study}

The obvious strengths of the URBAN Study are the: replication of international procedures and measures, neighborhood stratification and selection processes, use of objective and self-report measures, assessment of PA engagement over multiple domains, ability to control for seasonal effects, large sample size recruited, and incorporation of adults and children of diverse ethnicities into the sampling frame. Limitations of the study include its crosssectional research design that means causality cannot be determined, and that neighborhoods are only drawn from urban settings; therefore findings cannot be applied to rural or small town environments within New Zealand. Neighborhood walkability and ethnicity classifications may also differ by region, and communities classified as being higher walkable or higher Māori population in one city may not reach the inclusion threshold for another city. However, this may also be considered a strength of the study as the design will allow any city-specific or doseresponse effects to be captured, and assist with the understanding of the relative importance of other covariates and confounders. Lastly, neighborhoods are grouped according to geographic layout through contiguous meshblocks, rather than according to natural and social boundaries. This may create a mismatch between the GISassessed neighborhood and respondents' perceptions of their neighborhoods.

\section{Conclusion}

Taken together, the URBAN Study will generate robust scientific evidence by using appropriate and standardized measures to provide a New Zealand-specific understanding of the associations between urban design and health outcomes, as well as contributing data to an international research project. Providing this information will impart urgently needed epidemiological information regarding the associations between the built environment and health outcomes. Accordingly, this study directly addresses the international priority issues of increasing PA engagement and decreasing obesity levels at the population-level.

\section{Abbreviations}

A-NEWS: Abbreviated - Neighborhood Environment Walkability Scale; BMI: Body mass index; ${ }^{\circ} \mathrm{C}$ : Degrees Celsius; GIS: Geographical information systems; IPAQ-LF: International Physical Activity Questionnaire - Long Form; IPEN: International Physical Activity and Environment Network; mm: Millimeters; PA: Physical activity; SPACES: Systematic Pedestrian and Cycling Environment Scan; URBAN: Understanding the Relationships between Activity and Neighbourhoods.

\section{Competing interests}

The authors declare that they have no competing interests.

\section{Authors' contributions}

HMB developed the first draft of the manuscript. HMB, GMS, KW, PJS, SM, and RK contributed to the conception and the design of the study. All authors provided critical feedback during manuscript development. Each author has read and approved the final manuscript.

\section{Authors' information}

HMB is a Post-doctoral Research Fellow at the Centre for Physical Activity and Nutrition Research, Auckland University of Technology, New Zealand. GMS is a Professor of Public Health and the Director of the Centre for Physical Activity and Nutrition Research, Auckland University of Technology, New Zealand. KW is an Associate Professor at the Centre for Social and Health Outcomes Research and Evaluation, Massey University, New Zealand. PJS is a Professor of Biostatistics at the School of Public Health and Psychosocial Studies at Auckland University of Technology, New Zealand and the School of Nursing and Midwifery, University of Queensland, Australia. SM is a GIS Analyst at the Centre for Social and Health Outcomes Research and Evaluation, Massey University, New Zealand. RAK is a Professor of Geography at the School of Geography, Geology, and Environmental Sciences, University of Auckland, New Zealand. EAH is the Head of Research at the School of Sport and Recreation at Auckland University of Technology, New Zealand. MO is a Post-doctoral Research Fellow at the Centre for Physical Activity and Nutrition Research, Auckland University of Technology, New Zealand. HK is a researcher at Whariki Research Group, Massey University, New Zealand. VGJ is a researcher at Whariki Research Group, Massey University, New Zealand. CE is a PhD student at the School of Geography, Geology, and Environmental Sciences, Uni- 
versity of Auckland, New Zealand. LM is a PhD student at the Centre for Physical Activity and Nutrition Research, Auckland University of Technology, New Zealand. JM is the Research Manager at the Centre for Physical Activity and Nutrition Research, Auckland University of Technology, New Zealand

\section{Acknowledgements}

The URBAN Study is supported by a three-year research grant from the Health Research Council of New Zealand (grant: 07/356). The funding body was not involved in the design, conduct, data collection, management, or publication of the study. National Heart Foundation of New Zealand Research Fellowships support HMB and MO. The authors also gratefully acknowledge the participants who completed the study, research assistants who collect the data, and territorial authorities for providing the GIS datasets.

\section{References}

I. Ministry of Health: Healthy eating-healthy action: A background Ministry of Health: Wellington; 2003.

2. World Health Organization: Global strategy on diet, physical activity and health World Health Organization: Geneva; 2004.

3. World Cancer Research Fund and American Institute for Cancer Research: Food, Nutrition, Physical Activity, and the Prevention of Cancer: a Global Perspective American Institute for Cancer Research: Washington DC; 2007.

4. Sallis JF, Cervero RB, Ascher W, et al.: An ecological approach to creating active living communities. Ann Rev Public Health 2006 , 27:297-322.

5. Public Health Advisory Committee: Re-thinking urban environments and health Ministry of Health: Wellington; 2008.

6. Frank LD, Andresen MA, Schmid TL: Obesity relationships with community design, physical activity, and time spent in cars. Am J Prev Med 2004, 27:87-96.

7. Ewing R, Schmid TL, Killingsworth R, et al: Relationship between urban sprawl and physical activity, obesity and morbidity. Am J Health Prom 2003, 18:47-57.

8. Lopez-Zetina J, Lee H, Friis R: The link between obesity and the built environment. Evidence from an ecological analysis of obesity and vehicle miles of travel in California. Health \& Place 2006, I 2:656-664.

9. Owen N, Cerin E, Leslie E, et al:: Neighborhood walkability and the walking behavior of Australian adults. Am J Prev Med 2007 33:387-395.

10. Leslie E, Coffee N, Frank LD, et al:: Walkability of local communities: Using geographic information systems to objectively assess relevant environmental attributes. Health \& Place 2007 13:111-122.

II. Frank LD, Selens BE, Powell KE, et al.: Stepping towards causation: Do built environments or neighborhood and trave preferences explain physical activity, driving, and obesity? Soc Sci Med 2007, 65: I898-19|4.

12. Grow HM, Saelens BE, Kerr J, et al.: Where are youth active? Roles of proximity, active transport, and built environment. Med Sci Sports Exerc 2008, 40:207I-2079.

13. Holt NL, Spence JC, Sehn ZL, et al.: Neighborhood and developmental differences in children's perceptions of opportunities for play and physical activity. Health \& Place 2008, 14:2-14.

14. Badland HM, Schofield GM: Transport, urban design, and physical activity: An evidence based-update. Transportation Research Part D: Transport and Environment 2005, 10:177-196.

15. Sallis JF, Kraft K, Linton LS: How the environment shapes physical activity: a transdisciplinary research agenda. Am J Prev Med 2002, 22:208-2।I.

16. Eyler AA, Matson-Koffman D, Young DR, et al.: Quantitative study of correlates of physical activity in women from diverse racial/ethnic groups. Am J Prev Med 2003, 25(Suppl I):5-I4.

17. Giles-Corti B, Donovan RJ: Socioeconomic status differences in recreational physical activity levels and real and perceived access to a supportive physical environment. Prev Med 2002 35:60I-II.
18. Humpel N, Marshall AL, Leslie E, et al:: Changes in neighbourhood walking are related to changes in perceptions of environmental attributes. Ann Behav Med 2004, 27:60-67.

19. Ball K, Bauman AE, Leslie E, et al.: Perceived environmental aesthetics, convenience, and company are associated with walking for exercise among Australian adults. Prev Med 200I, 33:434-440.

20. King AC, Castro CC, Eyler AA, et al.: Personal and environmental factors associated with physical inactivity among different racial/ethnic groups of U.S. middle-aged and older-aged women. Health Psych 2000, 19:354-364.

21. Schlossberg M, Greene J, Paulsen Phillips P, et al:: School trips: Effects of urban form and distance on travel mode. J Am Planning Assoc 2006, 72:337-346.

22. Kerr J, Frank LD, Sallis JF, et al:: Urban form correlates of pedestrian travel in youth: Differences by gender, race-ethnicity and household attributes. Transportation Research Part D: Transport and Environment 2007, I 2: 177-182.

23. Hume C, Timperio A, Salmon J, et al.: Walking and cycling to school: Predictors of increases among children and adolescents. Am J Prev Med 2009, 36:195-200.

24. Epstein LH, Raja S, Gold SS, et al:: Reducing sedentary behavior: The relationship between park area and the physical activity of youth. Psych Sci 2006, 17:654-659.

25. Tudor-Locke $C$, Myers A: Challenges and opportunities for measuring physical activity in sedentary adults. Sports Med 200I, $31: 91-100$.

26. Welk G], Corbin CB, Dale D: Measurement issues in the assessment of physical activity in children. Res Q Exerc Sport 2000, 7 I (Suppl 2):59-73.

27. Ministry of Health: A portrait of health: Key results from the 2002/03 New Zealand Health Survey Ministry of Health: Wellington; 2004.

28. Sallis JF, Story M, Lou D: Study designs and analytic strategies for environmental and policy research on obesity, physical activity, and diet: Recommendations from a meeting of experts. Am J Prev Med 2009, 32(Suppl 2):72-77.

29. Statistics New Zealand: Census 2006 Statistics New Zealand: Wellington; 2007.

30. Frank LD, Sallis JF, Saelens BE, et al:: The development of the walkability index: Application to the Neighborhood Quality of Life Study. Br J Sports Med, Br J Sports Med 2009, 30:60I-6I I.

31. D'Sousa E, Forsyth A, Koepp J, et al.: Twin Cities walking study. Environment and physical activity: GIS protocols. Version 3.I Minneapolis: Metropolitan Design Center, University of Minnesota; 2006.

32. Statistics New Zealand: New Zealand in profile: An overview of new Zealand's people, economy, and environment Statistics New Zealand: Wellington; 2009.

33. Wang C, Burris M: Photovoice: Concept, methodology, and use for participatory needs assessment. Health Educ Behavior 1997, 24:367-387.

34. Wang C, Pies C: Family, maternal, and child health through photovoice. Matern Child Health J 2004, 8:95-102.

35. Pikora T], Giles-Corti B, Knuiman M, et al:: Neighborhood environmental factors correlated with walking near home: Using SPACES. Med Sci Sports Exerc 2006, 38:708-718.

36. Pikora TJ, Bull F, Jamrozik J, et al.: Developing a reliable audit instrument to measure the physical environment for physical activity. Am J Prev Med 2002, 23:187-194.

37. Puyau MR, Adolph AL, Vojra FA, et al.: Prediction of activity energy expenditure using accelerometers with children. Med Sci Sports Exerc 2004, 36:1625-1631.

38. Puyau MR, Adolph AL, Vojra FA, et al.: Validation and calibration of physical activity monitors in children. Obesity Res 2002, 10:150-157.

39. Welk GJ: Use of accelerometry-based activity monitors to assess physical activity. In Use of accelerometry-based activity monitors to assess physical activity Edited by: Welk GJ. Champaign: Human Kinetics; 2002:125-I42.

40. Craig CL, Marshall AL, Sjostrom M, et al.: International physical activity questionnaire: I2-country reliability and validity. Med Sci Sports Exerc 2003, 35: I38I-1395.

4I. Troiano RP, Berigan D, Dodd KW, et al:: Physical activity in the United States measured by accelerometer. Med Sci Sports Exerc 2008, 40: 181 - 188.

42. World Health Organization: The Asia-Pacific perspective: Redefining obesity and its treatment World Health Organization: Geneva; 2000. 
43. World Health Organization: Obesity and overweight World Health Organization: Geneva; 2006.

44. Cole TJ, Flegal KM, Nicholls D, et al.: Body mass index cut offs to define thinness in children and adolescents: An international study. BMJ 2007, 335:166-167.

45. World Health Organization: Measuring obesity: Classification and description of anthropometric data World Health Organization Regional Office for Europe: Copenhagen; 1988.

46. Hirschler V, Maccallini G, Calcagno M, et al.: Waist circumference identifies primary school children with metabolic syndrome abnormalities. Diab Tech Therap 2007, 9:149-I57.

47. Lean ME, Han TS, Morrison CE: Waist circumference as a measure for indicating need for weight management. BMJ 1995 , 3II:I58-16I.

48. Taylor RW, Williams SM, Grant AM, et al.: Waist circumference as a measure of trunk fat mass in children aged 3 to 5 years. Int J Ped Obes 2008, 3:226-233.

49. Cerin E, Saelens BE, Sallis JF, et al.: Neighborhood Environment Walkability Scale: validity and development of a short form. Med Sci Sports Exerc 2006, 38: I682-I69I.

50. Frank LD, Sallis JF, Conway TL, et al:: Many pathways from land use to health: Associations between neighborhood walkability and active transportation, body mass index, and air quality. J Am Planning Assoc 2006, 72:75-87.

51. Saelens BE, Sallis JF, Black JB, et al.: Neighborhood-based differences in physical activity: An environment scale evaluation. Am J Public Health 2003, 93: I552- I558.

52. Hoehner CM, Brennan Ramirez LK, Elliot MB, et al.: Perceived and objective environmental measures and physical activity among urban adults. Am J Prev Med 2005, 28(Suppl 2): I05-I I6.

53. Lovasi GS, Neckerman KM, Quinn JW, et al.: Effect of individual or neighborhood disadvantage on the association between neighborhood walkability and body mass index. Am J Public Health 2008, 99:279-284.

54. Nagel CL, Carlson NE, Bosworth M, et al:: The relation between neighborhood built environment and walking activity among older adults. Int J Epidemio 2008, I68:46 I-468.

55. Ball K, Jeffery RW, Crawford DA, et al: Mismatch between perceived and objective measures of physical activity environments. Prev Med 2008, 47:294-298.

56. Badland HM, Schofield GM, Schluter PJ: Objectively measured commute distance: Associations with actual travel modes and perceptions to place of work or study in Auckland, New Zealand. J Phys Act Health 2007, 4:80-86.

57. Leslie E, McCrea $R$, Cerin E, et al: Regional variations in walking for different purposes: The South East Queensland Quality of Life Study. Environ Behav 2007, 39:557-577.

58. Sallis JF, Saelens BE, Franks LD, et al.: Neighborhood built environment and income: Examining multiple health outcomes. Soc Sci Med 2009, 68: I285-1293.

\section{Pre-publication history}

The pre-publication history for this paper can be accessed here:

http://www.biomedcentral.com/1471-2458/9/224/pre pub
Publish with BioMed Central and every scientist can read your work free of charge

"BioMed Central will be the most significant development for disseminating the results of biomedical research in our lifetime. "

Sir Paul Nurse, Cancer Research UK

Your research papers will be:

- available free of charge to the entire biomedical community

- peer reviewed and published immediately upon acceptance

- cited in PubMed and archived on PubMed Central

- yours - you keep the copyright

Submit your manuscript here:

http://www.biomedcentral.com/info/publishing_adv.asp
BioMedcentral 